\title{
Flexural behaviour of corrugated panels of self-reinforced polypropylene hybridised with carbon fibre: An experimental and modelling study
}

\author{
Francisco Mesquita*, Anke van Gysel, Marina Selezneva, Yentl Swolfs, Stepan V. Lomov, \\ Larissa Gorbatikh
}

Department of Materials Engineering, KU Leuven, Kasteelpark Arenberg 44 bus 2450, Belgium

\section{A R T I C L E I N F O}

\section{Keywords:}

Hybrid

Polymer (textile) fibre

Finite element analysis (FEA)

Mechanical properties

\begin{abstract}
A B S T R A C T
The present work investigates the influence of the laminate layup on the flexural properties and failure mechanisms of corrugated panels made of self-reinforced polypropylene (SRPP) hybridised with carbon fibre. The influence of the layup on the stiffness and strength of the corrugated hybrid composite panels was much less important than the carbon fibre content. The experimental program was complemented by finite element analysis. The model revealed the importance of SRPP's non-linear behaviour and difference in tensile and compressive behaviour in controlling the flexural properties of corrugated panels. The compressive behaviour of SRPP was characterised to provide reliable input data for the model.
\end{abstract}

\section{Introduction}

Self-reinforced composites (SRCs) outperform conventional polymers as they are reinforced with oriented polymeric chains, giving way to higher stiffness and strength while still offering higher failure strain [1]. Among SRCs, self-reinforced polypropylene (SRPP) has attracted the most attention thanks to its combination of high service temperature and toughness, wider processing window and low price compared to other SRCs [2,3]. The use of SRPP in applications involving flexural loads is not straightforward as its compressive strength is significantly lower than the tensile strength as revealed in previous research on unidirectional [4] and woven [5] SRPP. This explained why specimens tested in flexure always failed on the compressive side while tensile and flexural moduli were found to be the same [6].

SRPP's stiffness and strength are still relatively low in comparison with fibre reinforced plastics like carbon or glass fibre composites, having limited its use in structural applications [2,7]. By hybridising SRPP with carbon fibres, a new composite can be designed with improved overall performance, including flexural properties. Hybridisation has been used as a strategy to increase the flexural properties of composites. For example, the flexural strength of carbon fibre composites was shown to increase by replacing the carbon fibres on the compressive side by glass or silicon carbide fibres [8,9]. The flexural properties of hybrid composites do not only change with the constituent properties but also with the laminate layup $[10,11]$. If the stiffer layers are further away from the neutral plane of the laminate, their contribution to the stiffness and strength is higher.

When using corrugated panels, the distance between the layers and the neutral plane is larger than the thickness of the panel (see Fig. 1). Corrugation therefore increases the flexural stiffness of the panel made of any material. These corrugated structures are very stiff in the longitudinal direction, but they remain relatively compliant in the transverse direction [12] (see Fig. 1). Properly designing the materials used in a corrugated panel is fundamental for their effective use.

The classical laminate theory (CLT) is a useful tool for the design of composite corrugated panels. Analytical equations have been derived following the CLT approach to convert a corrugated structure into an orthotropic plate $[12,13]$. Another approach was taken in [14] where the panel was divided into flanges and webs (see Fig. 1). The authors derived an apparent modulus of a corrugated panel which takes into account the material properties and the cross-sectional shape. For nonlinear materials such as SRPP, the applicability of the CLT is limited. It can still be used for tensile loading conditions [15] but not for flexural as the stress varies along the length of the panel. Contrary to CLT, finite element (FE) methods can be used to capture highly non-linear materials flexural behaviour such as that of SRPP.

The current study aims to provide guidelines for the design of corrugated panels made of hybrid SRPP laminates loaded in flexure. Manufacturing SRPP in an inherently stiffer corrugated geometry provides opportunities for broadening SRPP's potential use in higher load carrying applications. The layup of the hybrid corrugated panel is varied to assess its influence on the flexural properties and failure

\footnotetext{
* Corresponding author.

E-mail address: francisco.mesquita@kuleuven.be (F. Mesquita).
} 


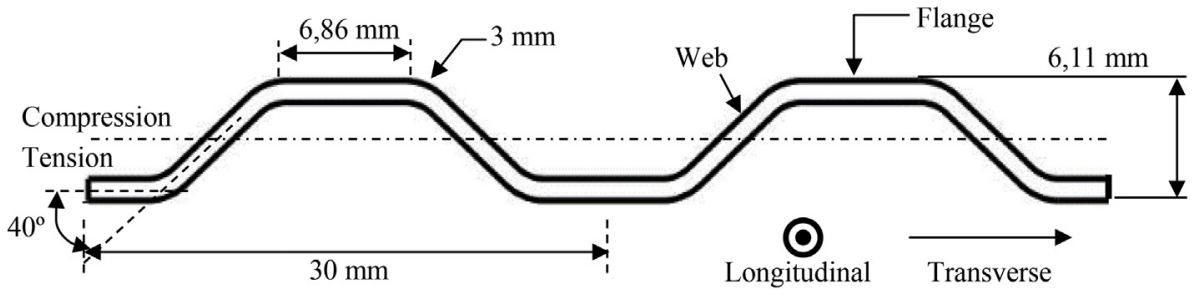

Fig. 1. Geometry and dimensions of the corrugated panel. The corrugated panel's cross section is trapezoidal with round edges. mechanisms. An FE model is developed to help the interpretation of the experimental results. The model predicts the mechanical behaviour of the corrugated panels. The compressive properties of SRPP are less investigated in the literature and are measured in the current study to support the modelling work.

\section{Materials and methods}

\subsection{Materials}

Propex Fabrics GmbH (Germany) provided polypropylene (PP) tapes with a width of $2.4 \mathrm{~mm}$ and woven into a twill 2-2 pattern. Prior to hot compaction, the tapes have a tensile strength, modulus and failure strain of $500 \mathrm{MPa}, 10 \mathrm{GPa}$ and $9 \%$ respectively [16]. After compaction, the woven layers have a thickness of $150 \mu \mathrm{m}$. Propex Fabrics GmbH also provided PP films with a thickness of $20 \mu \mathrm{m}$ and consisting of the same polymer grade as the tapes. Unidirectional carbon fibre reinforced polypropylene (UD CFRPP) prepregs were sourced from Jonam Composites (United Kingdom). The prepregs contain T700 carbon fibres but the exact grade of polypropylene is not known. Prior to hot compaction, the prepregs have a thickness of $160 \mu \mathrm{m}$ and a carbon fibre volume fraction of $32 \pm 1 \%$.

\subsection{Hot compaction}

The corrugated panels were manufactured using a Pinette Press Zenith 2. Layers of woven SRPP and UD CFRPP prepregs were stacked between two halves of a polished aluminium mould with the shape of the corrugated panel (see Fig. 1). No PP films were used in the production of the corrugated panels. The hot compaction was performed at $188^{\circ} \mathrm{C}$ and $39 \mathrm{bar}$ for $5 \mathrm{~min}$ [17]. The cooling rate was $5^{\circ} \mathrm{C} / \mathrm{min}$ on average. The processing temperature has a minor influence on the tensile modulus and strength between temperatures of $184-191^{\circ} \mathrm{C}$ as demonstrated in [17]. The press was not pre-heated prior to the manufacturing as that would imply heating the thick mould as well.

During the hot compaction process, the PP in the CFRPP prepregs had the tendency to flow out of the mould. The result was that the thickness of the carbon fibre layers was reduced from 160 to $100 \mu \mathrm{m}$, which was confirmed later with the use of optical microscopy. The SRPP layers maintained the nominal thickness of $150 \mu \mathrm{m}$. The panel's thickness was not homogenous. By performing thickness measurements, it was observed that the real panels had a higher thickness in the webs oriented in one direction than on the other webs, as shown in Fig. 2. The influence of the non-uniform thickness is further discussed in section 3.3.1.

To produce specimens for compression testing, 25 layers of woven PP-tapes were stacked between flat aluminium plates. One polypropylene film of $20 \mu \mathrm{m}$ was placed between each two layers of SRPP to assure good adhesion between layers. A Fontijne Grotnes LabPro 400 press was used for the hot compaction of these specimens using the same parameters as for the corrugated panels described above. The plates were then cooled down to $40^{\circ} \mathrm{C}$ in $5 \mathrm{~min}$. For these panels, the press was first pre-heated up to the compaction temperature to ensure a homogenous temperature distribution.

\subsection{Specimen description}

\subsubsection{Corrugated panels}

The basic geometry of the corrugated panel is trapezoidal with round edges (see Fig. 1). The length and width of the panel were 310 and $120 \mathrm{~mm}$, respectively. The mould was designed for a nominal thickness of $1,11 \mathrm{~mm}$, making the use of 7 layers in total appropriate. The stacking sequences were restricted to the ones where SRPP was the outmost layer for surface quality reasons. Imposing the additional constraint of using only symmetrical layups leaves 8 layups possible. The layups studied and their $\mathrm{CF}$ volume fraction $\left(\mathrm{V}_{\mathrm{f}}\right)$ are presented in Table 1. To determine the $\mathrm{CF} \mathrm{V}_{\mathrm{f}}$ of each lay-up, equation (1) was used,

$V_{f}=\frac{n^{C} \cdot t^{C} \cdot V_{f}^{C}}{n^{C} \cdot t^{C}+n^{S} \cdot t^{S}}$

where $n^{C}$ is the number of UD CFRPP layers, $n^{S}$ is the number of SRPP layers, $t^{C}$ is the initial thickness of the UD CFRPP prepregs, $t^{S}$ is the thickness of the SRPP layers and $V_{f}{ }^{C}$ is the carbon fibre volume fraction within a single layer of UD CFRPP. The letters "S" and "C" in the layup annotation in Table 1 stand for SRPP and UD CFRPP, respectively. The "so" outside the parenthesis means that the lay-up is symmetrical and contains an odd number of layers. In this case, the fourth letter represents the middle layer. The thickness values were measured in the mid-span line on the extremities of the panel along the width. These values are approximate as it was also verified that the thickness is not homogenous along the panel (see Fig. 2).

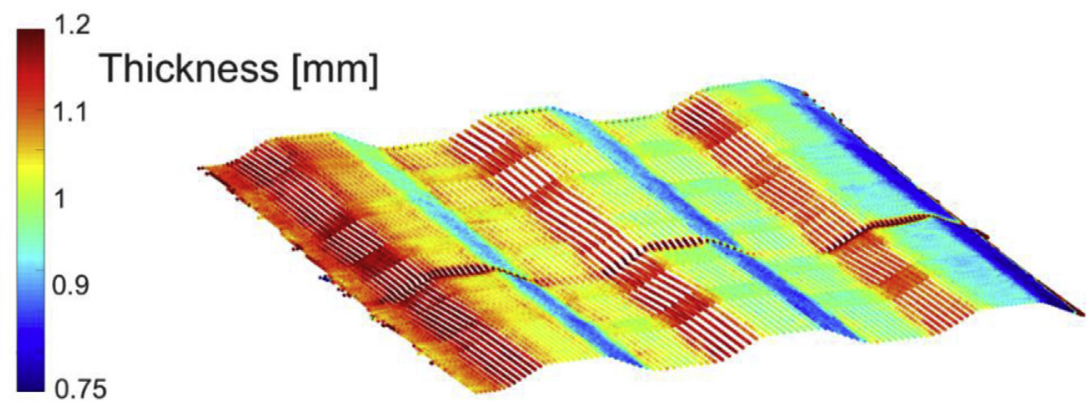

Fig. 2. 3D coordinate measurements on a part of a tested hybrid corrugated panel. The thickness is higher in the webs oriented to one of the sides than on the others. The panel is generally thicker on the left side of the image. 
Table 1

Stacking sequences of the corrugated hybrid panels produced and tested. The thickness was measured in 4 points along the middle of the span.

\begin{tabular}{ccc}
\hline Stacking Sequence & CF V $_{\mathrm{f}}$ & Thickness [mm] \\
\hline$(S / S / S / S)_{s o}$ & $0 \%$ & $1.05 \pm 0.10$ \\
$(S / S / S / C)_{s o}$ & $5 \%$ & $1.00 \pm 0.30$ \\
$(S / S / C / S)_{s o}$ & $10 \%$ & $0.95 \pm 0.01$ \\
$(S / C / S / S)_{s o}$ & $10 \%$ & $0.96 \pm 0.02$ \\
$(S / S / C / C)_{s o}$ & $14 \%$ & $0.88 \pm 0.02$ \\
$(S / C / S / C)_{s o}$ & $14 \%$ & $0.89 \pm 0.02$ \\
$(S / C / C / S)_{s o}$ & $19 \%$ & $0.83 \pm 0.03$ \\
\hline
\end{tabular}

\subsubsection{Compression test specimens}

The compressive behaviour of SRPP depends on the processing conditions as the compaction influences the layer bonding. The aspect ratio can also influence the results due to the possibility of buckling. To ensure a broad understanding of the influence of the geometry of the specimen, the width was varied. The thickness was kept constant at $4,25 \pm 0,06 \mathrm{~mm}$.

ASTM D3410 requirements were used to determine the gage length $l_{g}$, of the specimens. The specimens had to be long enough to allow that the stress concentrations imposed by the grips were limited, but also short enough to avoid Euler buckling. The lower and upper limits to the gage length are shown in Table 2 for each specimen configuration. There is a conflict between the upper and lower limits of gage length for the 8 and $15 \mathrm{~mm}$ wide specimens. To ensure that the specimen did not buckle, the gage length was kept shorter than the upper limit. Two different gage lengths were chosen to study the effect of this dimension.

\subsection{Test methods}

\subsubsection{Flexural tests}

Three point bending tests on the corrugated panels (see Fig. 3) were performed according to ASTM D7264 - 07 [18] on an Instron 5567 equipped with a $1 \mathrm{kN}$ load cell. The span length used was $250 \mathrm{~mm}$, which corresponds to a span to height ratio of about 40 , well within the range suggested in the standard. At least five specimens from each layup were tested. The loading nose displacement rate was set to $10 \mathrm{~mm}$ / $\mathrm{min}$. The rollers used were $150 \mathrm{~mm}$ long and $40 \mathrm{~mm}$ in diameter. Since none of the samples fractured, the tests were stopped when the load dropped from the maximum value and then reached a plateau.

The application of a flexural load to a corrugated structure tends to change its shape until it becomes flat. At least one corrugated sample from each lay-up was monitored during the test using a high resolution camera. The camera monitored the edge of the panel in the middle of the span (see Fig. 3). With the images taken and processed by ImageJ, it was possible to measure the height of the cross section during the test.

\subsubsection{Optical microscopy}

After the flexural tests, the visibly damaged locations in the corrugated panels were cut out and analysed under an inverted light optical microscope Leica DL ILM HC. The images were captured with a digital camera Coolpix 990. The cut specimens and plane of observation are presented in Fig. 4. The specimens were cut from the mid-span region,

Table 2

Compression test specimen configurations and gage length limits.

\begin{tabular}{ccccc}
\hline Width [mm] & $\begin{array}{c}\text { Lower limit } \boldsymbol{l}_{\boldsymbol{g}} \\
{[\mathrm{mm}]}\end{array}$ & $\begin{array}{c}\text { Upper limit } \boldsymbol{l}_{\mathrm{g}} \\
{[\mathrm{mm}]}\end{array}$ & $\boldsymbol{l}_{\boldsymbol{g}}[\mathrm{mm}]$ & $\begin{array}{c}\text { Number of } \\
\text { specimens }\end{array}$ \\
\hline \multirow{2}{*}{4} & 13.5 & 21.55 & 15 & 3 \\
& & & 18 & 2 \\
8 & 27.02 & 21.55 & 15 & 3 \\
& & & 18 & 2 \\
15 & 50.65 & 21.55 & 18 & 3 \\
\hline
\end{tabular}

where the load is maximal both from the tension and compression loaded sides. A reference specimen was cut from an undamaged region.

The cut specimens were then embedded in epoxy resin to better stabilise the specimen position. The resin embedded specimens were grinded and polished using rotary abrasive disks to ensure good image quality.

\subsection{3. $3 D$ coordinate measurement}

The corrugated panels' thickness was measured using a COORD3 MC16 3D CMM. The device was used with optical scanning using a Nikon metrology laser line scanner LC60Dx. The maximum permissible error of the scanner is $9 \mu \mathrm{m}$. A point grid was determined from the surface of each side of the panel. Knowing the coordinates of the points on both sides of the panel, the thickness distribution could be determined.

\subsubsection{Compression tests}

The tests were conducted on an Instron 5567 compression machine equipped with a $30 \mathrm{kN}$ load cell and mechanical grips. Sandpaper was used in between the specimens and the grips to avoid slippage. The loading rate was $2 \mathrm{~mm} / \mathrm{min}$. Digital image correlation (DIC) was performed to measure the average surface strain. A speckle pattern was applied on the surface and the back and front surface were tracked by two cameras. This approach made it possible to measure the difference in strains and to detect out-of-plane deformation. Buckling can happen in the image plane (in-plane) or in the direction normal to the image (out-of-plane). The standard states that buckling occurs if the percentage of bending $B_{y}$ (Equation (2)) in the specimen reaches a value higher than $10 \%$ [19].

$\boldsymbol{B}_{\boldsymbol{y}}=\frac{\left|\varepsilon_{1}-\varepsilon_{2}\right|}{\varepsilon_{1}+\varepsilon_{2}} \cdot 100$

The strains $\varepsilon_{1}$ and $\varepsilon_{2}$ are measured on the front and back side of the specimen, respectively.

\subsection{Modelling approach}

\subsubsection{FE model}

A FE model was developed to predict the flexural properties of a hybrid corrugated panel using ABAQUS 2016. The design of the panel is identical to the one described in section 2.3.1. As in the experimental setup, the span length was $250 \mathrm{~mm}$ so that comparisons could be made. Symmetry conditions were applied to the mid-span and to one of the side faces, enabling the reduction of the model to one quarter of the panel. To avoid numerical problems and study only the influence of the material properties on the panel, contact was not simulated. Instead, the displacement was applied directly on the contact areas between the loading nose and the corrugated panel through the use of a reference point. The reference point was constrained to these areas in a way that all the displacements applied to the reference point would be applied to the corrugated panel. Through this method, the total reaction force could be measured at the reference point. In the support roll contact region, the displacement in the loading direction was inhibited. The panel design and the boundary conditions are illustrated in Fig. 5.

The model was built with C3D8R elements, which are 8-noded bricks with reduced integration and hourglass control. Each layer contained 2 elements in the thickness direction, corresponding to 14 elements along the panel's thickness. A global element size was applied in all other directions, with an approximate size of $0.5 \mathrm{~mm}$. Each model contained approximately 600.000 elements. No cohesive elements were used in the model, thus imposing that delamination could not occur.

\subsubsection{Materials properties}

The elastic properties of SRPP were obtained using the software packages WiseTex and TexComp with the detailed geometry and properties of the SRPP tapes described in section 2.1 and complemented 


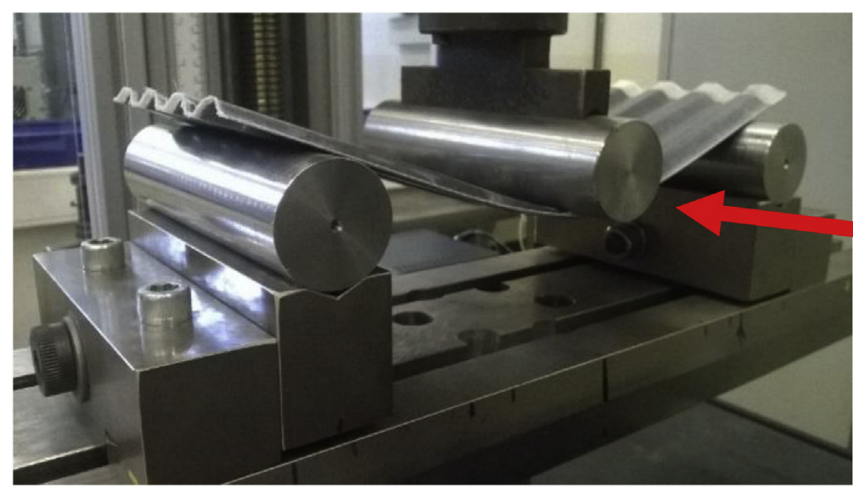

Camera direction

Fig. 3. Three-point bending test on a corrugated panel and the position of the camera to record the global deformation of the panel in the mid-span region.

with the information provided in [20] for SRPP tapes. Table 3 summarises the SRPP weave properties. The tape dimensions in the compacted SRPP was adapted so that the material's stiffness would coincide with its experimentally measured value of $3.04 \mathrm{GPa}$. The elastoplastic behaviour of SRPP was taken into account in the FE model. As SRPP has a bi-linear tensile and compressive behaviour, the second part of stressstrain curves was obtained by introducing the experimental data under the plastic domain of the material in ABAQUS 2016. The top and bottom sides of the corrugated panel are under tension or compression, which was taken into account assigning corresponding SRPP properties depending on the load condition.

The UD CFRPP was considered to be fully elastic. The elastic properties of T700 fibres used were based on data from [21-23]. The polypropylene matrix was characterised by a stiffness of $1,5 \mathrm{GPa}$ and a Poisson's ratio of 0.3. Chamis' [24] formulae were then used to obtain the elastic properties of UD CFRPP. The elastic constants of all materials used are presented in Table 4.

\section{Results and discussion}

\subsection{Flexural tests}

Since the standard is designed for specimens with rectangular cross section specimens, it is not possible to determine a stress-strain curve of the corrugated panels. The force-displacement curves were therefore used to determine the flexural stiffness and maximum flexural load. Due to the thickness variability between layups, the properties were divided by the thickness of each panel, achieving a normalised stiffness and maximum load.

\subsubsection{Flexural properties}

The flexural stiffness was measured in the linear part of the force displacement curve, which occurred between 1 and $3 \mathrm{~mm}$ of the loading nose displacement. In all of the panels, the maximum flexural load was followed by a gradual load drop but without complete fracture. Fig. 6 presents the stiffness and maximum load variation with the stacking sequence. The modelling results are discussed in section 3.3.1.

The stiffness of the corrugated panels increases with the carbon fibre content and with the relative distance between the UD CFRPP layer and the neutral axis. The layup variation hardly changes the distance from the UD CFRPP layers to the neutral axis. The influence of the layup variation should therefore be much higher in flat specimens than in the corrugated panels.

The maximum flexural load also increases with the $C F V_{f}$ but the trend is less pronounced. The $(\mathrm{S} / \mathrm{S} / \mathrm{C} / \mathrm{C})_{\text {so }}$ layup has a higher $\mathrm{CF} \mathrm{V}_{\mathrm{f}}$ than the $(\mathrm{S} / \mathrm{C} / \mathrm{S} / \mathrm{S})_{\text {so }}$ layup but they show the same maximum flexural load. The same happens between the $(\mathrm{S} / \mathrm{C} / \mathrm{C} / \mathrm{S})_{\text {so }}$ and $(\mathrm{S} / \mathrm{C} / \mathrm{S} / \mathrm{C})_{\text {so }}$ layups. The $(\mathrm{S} / \mathrm{S} / \mathrm{C} / \mathrm{C})_{\text {so }}$ and $(\mathrm{S} / \mathrm{C} / \mathrm{C} / \mathrm{S})_{\text {so }}$ have CFRPP layers stacked together, decreasing the panel's maximum load. When a critical carbon fibre break cluster develops in one of the layers, it propagates to the other, failing both carbon layers simultaneously. The (S/C/S/S) so and (S/C/S/ C) $)_{\text {so }}$ layups have carbon fibre layers stacked between SRPP layers, allowing independent failure of the carbon fibre layers.

Fig. 7 shows the measured force-displacement curves for all the layups. The hybrid composite panel with a single carbon fibre layer has a flexural stiffness which is by a factor of three higher than that of the pure SRPP panel. Its toughness (area under the load-displacement curve) is still high as the load drops gradually. In all other hybrid composite panels, the flexural stiffness and maximum load are higher but their toughness is lower.

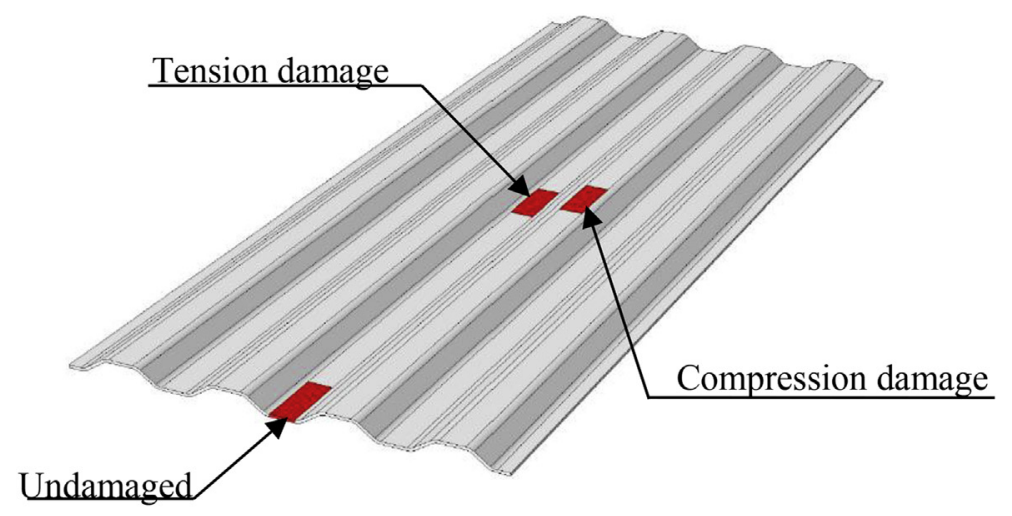

(a)

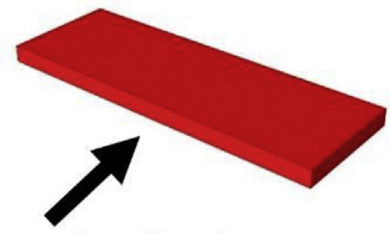

Observation direction

Fig. 4. (a) Specimen cut location and (b) plane of observation in microscopy control. 


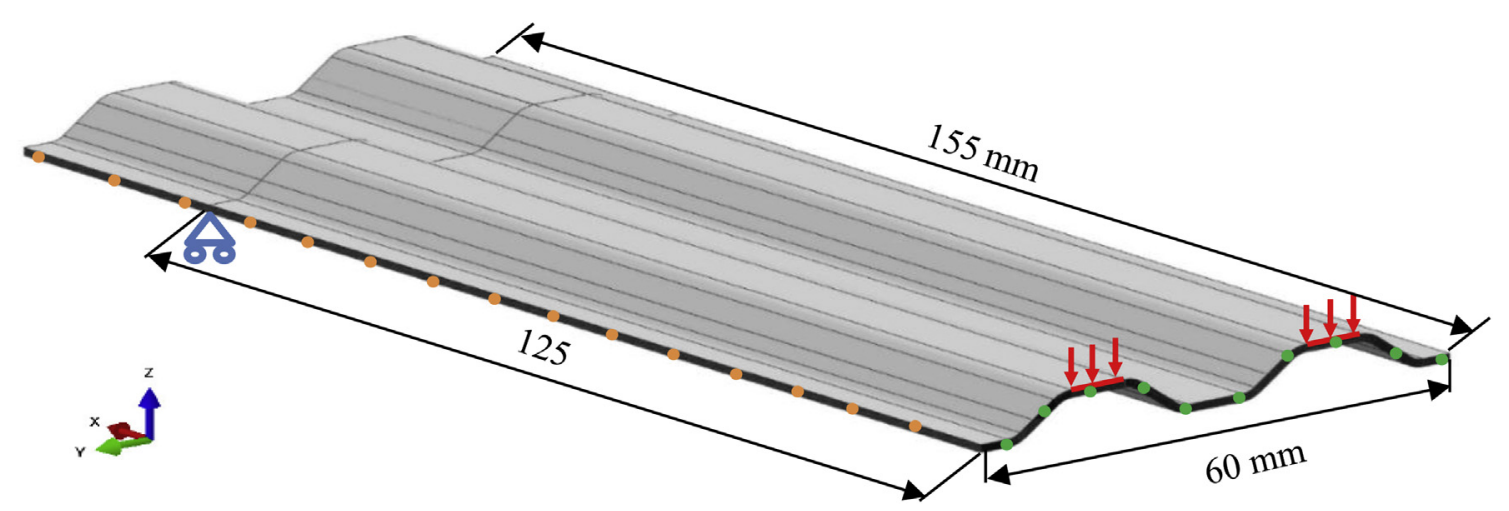

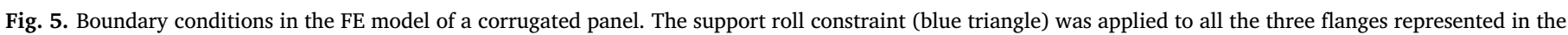

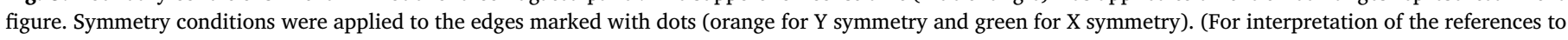
colour in this figure legend, the reader is referred to the Web version of this article.)

Table 3

Summary of the SRPP weave properties.

\begin{tabular}{|c|c|c|c|c|c|}
\hline \multirow[t]{2}{*}{ Tape } & Tensile modulus [GPa] & Tensile strength [MPa] & Failure strain [\%] & Thickness [mm] & Width [mm] \\
\hline & 10 & 500 & 9 & 0.05 & 2.0 \\
\hline \multirow[t]{2}{*}{ Weave } & Weave architecture & Areal Density $\left[\mathrm{g} / \mathrm{m}^{2}\right]$ & Compacted Thickness [mm] & Warp/Weft count [yarns/cm] & Tape volume fraction [\%] \\
\hline & Twill-2/2 & 140 & 0.15 & 6.37 & 75 \\
\hline
\end{tabular}

\section{Table 4}

Elastic properties of the materials used in the model. The directions 1 and 2 are the in plane directions for the fabric/prepregs. For the prepregs, direction 1 corresponds to the fibres longitudinal direction. For both cases, 3 corresponds to the thickness direction.

\begin{tabular}{ccc} 
& SRPP & UD CFRPP \\
\hline $\mathrm{E}_{11}[\mathrm{GPa}]$ & 3.04 & 74.62 \\
$\mathrm{E}_{22}[\mathrm{GPa}]$ & 3.04 & 3.06 \\
$\mathrm{E}_{33}[\mathrm{GPa}]$ & 1.38 & 3.06 \\
\hline $\mathrm{v}_{12}$ & 0.15 & 0.36 \\
$\mathrm{v}_{13}$ & 0.26 & 0.36 \\
$\mathrm{v}_{23}$ & 0.26 & 0.30 \\
\hline $\mathrm{G}_{12}[\mathrm{GPa}]$ & 0.71 & 3.19 \\
$\mathrm{G}_{13}[\mathrm{GPa}]$ & 0.47 & 3.19 \\
$\mathrm{G}_{23}[\mathrm{GPa}]$ & 0.47 & 1.18 \\
\hline
\end{tabular}

\subsubsection{Failure analysis}

All the specimens failed on the compression side. When damage appears on SRPP due to tape debonding, matrix cracking, delamination, etc., the material becomes white [25]. That was evident from the apparition of white transverse lines on the panel surface in contact with the loading nose. When observed under the microscope, the main failure mechanism in the $(\mathrm{S} / \mathrm{S} / \mathrm{S} / \mathrm{S})_{\text {so }}$ specimens was tape debonding, which correlates with the whitening of the material under compression (Fig. 8a). In the hybrid layups, the CF layers broke and triggered delamination between the UD CFRPP and SRPP layers (Fig. 8b).

Delamination caused by the poor adhesion between layers was the main failure mechanism observed in the experiments. On one hand, this phenomenon can be useful if it occurs gradually as it can increase the toughness of the panel. On the other hand, if it occurs suddenly and catastrophically, it will reduce the load carrying capacity of the material after delamination occurs. The consistent failure on the compressive side of the panels evidences the lower strength of SRPP under compressive loads. This was verified by the results presented in the following section.

\subsection{Compression tests}

The results of the compression tests on SRPP specimens can be found on Table 5. The table displays the strength, modulus and failure strain as a function of the specimen geometry. The gage length differences did not influence the results ( $p$-value is $80 \%$ ), enabling the grouping of the results for each cross-section. For comparison, the last row of the table shows the properties of SRPP under tensile loading [26]. Fig. 9 shows the stress-strain curves of the specimens mentioned in this section, grouped by configuration.

The compressive strength proved to be lower than the tensile strength for all the specimens tested. The compressive and tensile modulus were approximately the same for the $4 \times 8 \mathrm{~mm}$ and $4 \times 15 \mathrm{~mm}$ specimens but not for the $4 \times 4$ specimens (see Fig. 9). The smallest specimens were difficult to align and thus in-plane buckling was prone to occur. One of the smallest specimens achieved significantly higher compressive strength, modulus and failure strain than the others. This "outlier" specimen was probably better aligned with the loading direction since the limit percentage of buckling (see Equation (2)) was only reached close to the final failure.

The results from the compression tests explain why all the corrugated panels failed on the compressive side. Since the elastic modulus is similar for both loading conditions, the regions loaded in tension and compression in the corrugated panels behave equally in the beginning of the test. If the elastic properties were not the same, the neutral axis would not be in the middle of the panel (see Fig. 1). In that case, the modelling approach of dividing the panel into its compressive and tensile side would not have been correct (see section 2.5.1).

\subsection{Modelling}

\subsubsection{Load-displacement diagrams}

The flexural behaviour of the panels was modelled for all of the stacking sequences tested. The displacement applied in the simulation was the same as the displacement at the maximum flexural load observed for each layup. This was the best approach possible as the main failure mechanisms were not modelled. Fig. 6 presents the comparison 


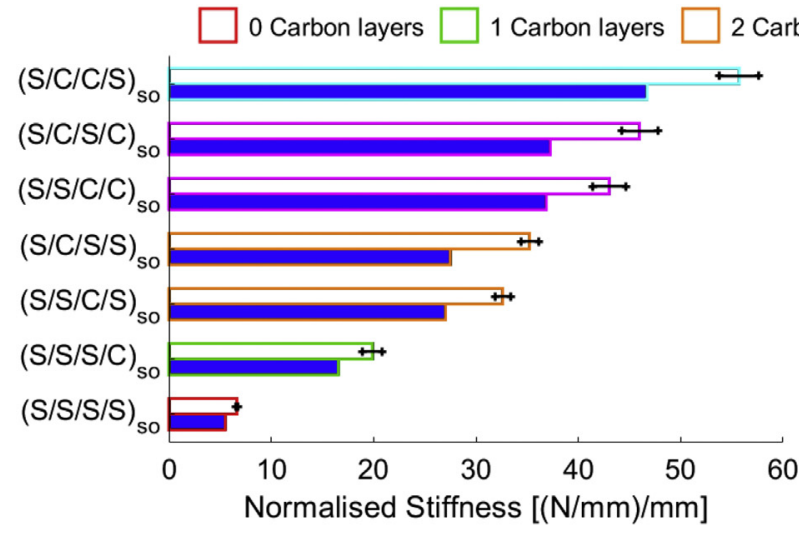

(a)

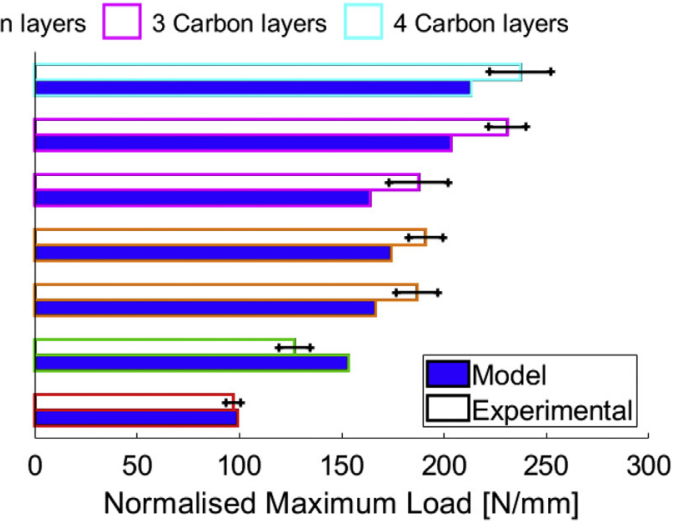

(b)

Fig. 6. Measured and predicted flexural properties of the hybrid corrugated panels: (a) stiffness and (b) maximum load, both normalised to the panel thickness. The predicted stiffness is consistently lower than the measured one. The modelled stiffness is not sensitive to the CF layer position.

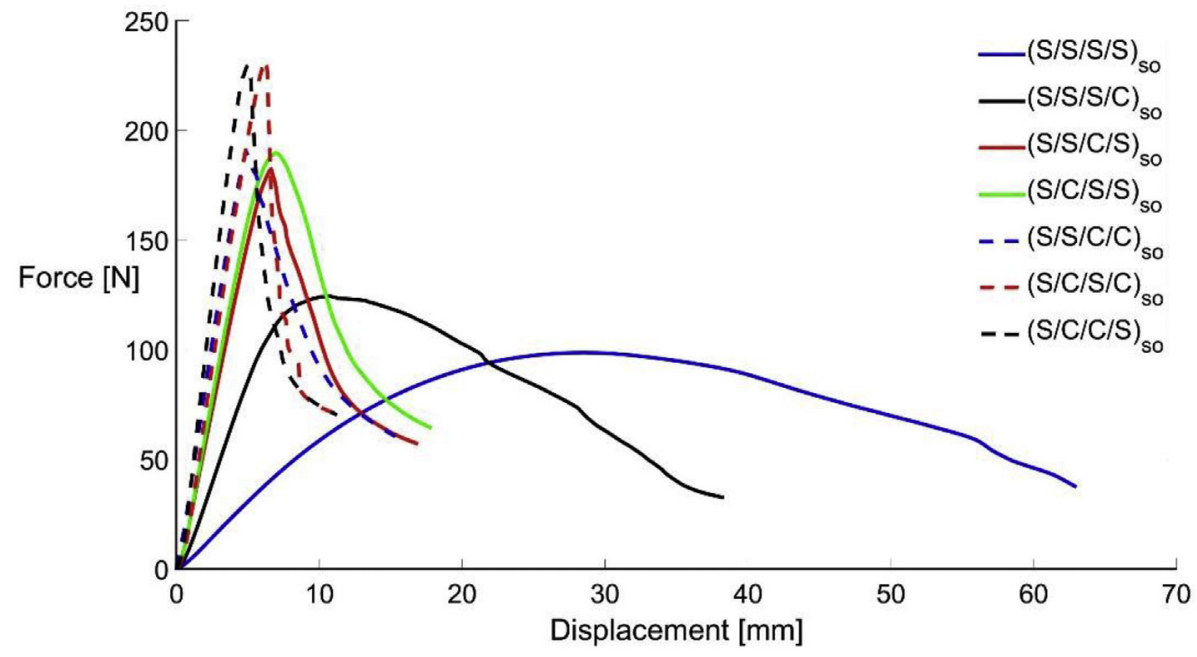

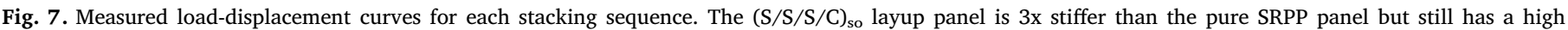
toughness.

between the model and experiments for the stiffness and maximum flexural load. The results were normalised to the panel thickness to allow for a fair comparison.

The modelled stiffness was always lower than the experimental value. As mentioned in section 2.2, the thickness of the real panels is not homogenous (see Fig. 2). The thickness of the carbon fibre layers also decreased during production of the panels but the volume fraction may have increased. Finally, the input parameters for SRPP's tensile behaviour in the model were based on tests where SRPP was produced using PP films. The corrugated panels were produced without films. These three factors may explain why the modelled stiffness is systematically lower than the experimentally measured.

Although there was a difference in stiffness, the maximum flexural load showed good agreement between experiments and the model (see Fig. 6b). The experimental force-displacement curves revealed a nonlinearity close to the maximum load point, whereas that non-linearity did not occur in the model, which can be attributed to the absence of damage in the model.

The distinction between the tensile and compressive properties of SRPP made a significant difference in the results for the pure SRPP panel. Even though SRPP is stronger in tension than in compression, the force supported under compression for low strains was higher. This effect is clear in Fig. 9, where it is shown that the compressive stress is higher than the tensile stress for the same strain level. The panel therefore carried higher loads in the model assuming the different behaviour under tension and compression than if all the material had the tensile behaviour (see Fig. 10).

\subsubsection{Mid-span deformation}

The height of the cross section of the corrugated panel tended to decrease during the test and its profile tended to flatten. The measurement of the panel height during the flexural test is described in section 2.4.4. The same measurement can be performed in the model, providing further support for the accuracy of the results. The height variation was normalised so that the initial relative height was 1 and all the other values are a fraction of the initial value.

The results shown in Fig. 11a are for the $(\mathrm{S} / \mathrm{S} / \mathrm{S} / \mathrm{S})_{\text {so }}$ panel and confirm the accuracy of the model. For all the other specimens, the black carbon fibre layers hampered the height reduction measurements due to the lack of contrast against the background. As load is applied, the flanges on each side of the panel start rotating (Fig. 11b), creating uncertainty as to where the extreme points of the panel are. The initial height variation of the hybrid specimens nevertheless agrees with the modelling predictions. 


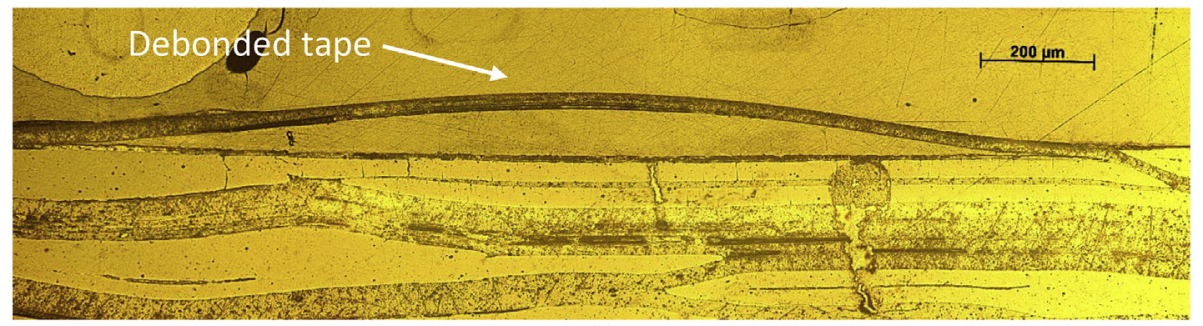

(a)

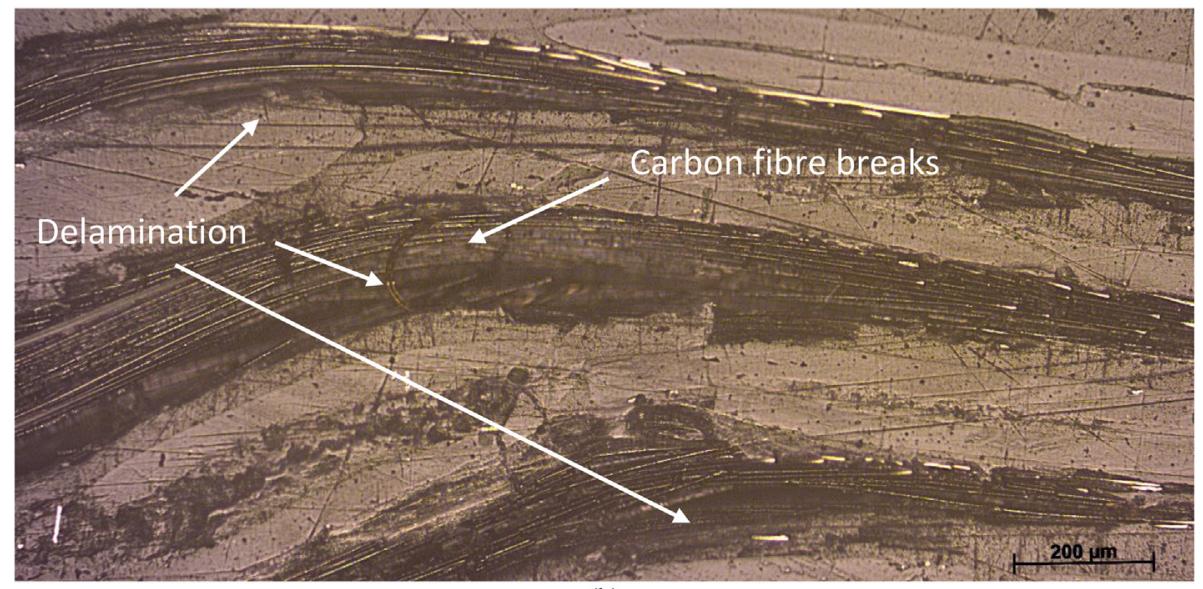

(b)

Fig. 8. (a) Tape debonding at the surface of the $(\mathrm{S} / \mathrm{S} / \mathrm{S} / \mathrm{S})_{\text {so }}$ corrugated panel. (b)Broken carbon fibre layers and delamination in a (S/C/S/C) so panel.

Table 5

Compression and tensile test results for SRPP [26].

\begin{tabular}{lcccc}
\hline & $\begin{array}{c}\text { Width } \\
{[\mathrm{mm}]}\end{array}$ & Strength [MPa] & $\begin{array}{c}\text { Elastic modulus } \\
{[\mathrm{GPa}]}\end{array}$ & Failure strain [\%] \\
\hline Compression & 4 & $36.6 \pm 2.1$ & $2.17 \pm 0.17$ & $4.72 \pm 0.35$ \\
& 8 & $50.8 \pm 2.1$ & $2.91 \pm 0.21$ & $5.64 \pm 0.22$ \\
& 15 & $51.9 \pm 2.9$ & $2.80 \pm 0.11$ & $5.50 \pm 0.04$ \\
\hline Tension [26] & & $154.1 \pm 3.6$ & $3.06 \pm 0.05$ & $19.46 \pm 1.26$ \\
\hline
\end{tabular}

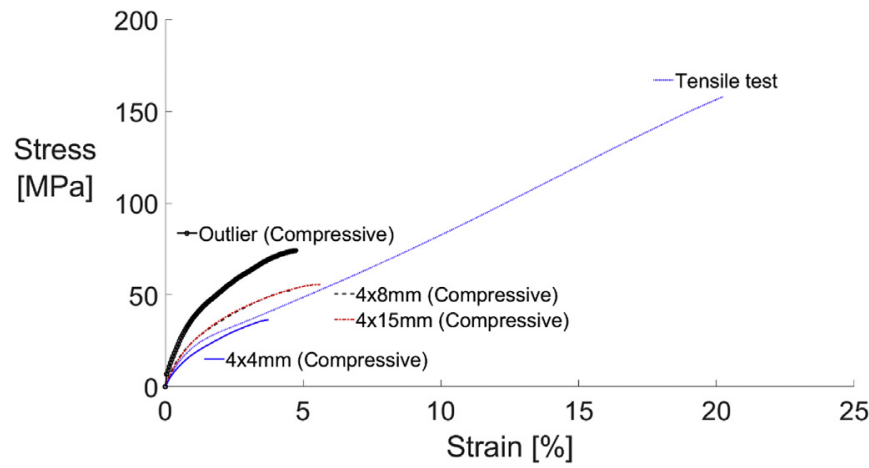

Fig. 9. Tensile and compressive behaviour of SRPP. The outlier specimen was the strongest from the compression test specimens. SRPP is weaker in compression than in tension.

\section{Conclusion}

The flexural stiffness of hybrid composite corrugated panels is strongly influenced by the hybrid volume fraction but the CF layer position in the layup is less important. The stiffness variation with the $\mathrm{CF}$ layer position is expected to be higher in flat specimens. The maximum load also increases with the CF volume fraction. However, for layups where the UD CFRPP layers were stacked together, the layer

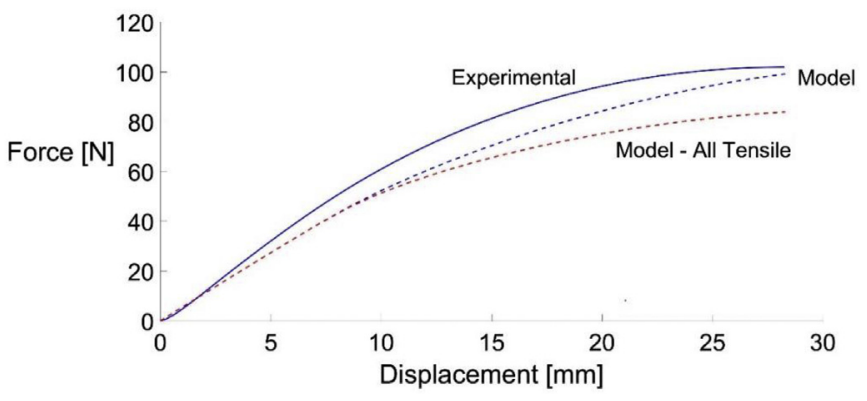

Fig. 10. Experimental and modelling force displacement curves for a pure SRPP corrugated panel. The blue dashed curve shows the results using the compressive properties of SRPP while the red curve only uses the tensile properties. Using SRPP's compressive properties in the FE model increases the correlation with the experimental results. (For interpretation of the references to colour in this figure legend, the reader is referred to the Web version of this article.)

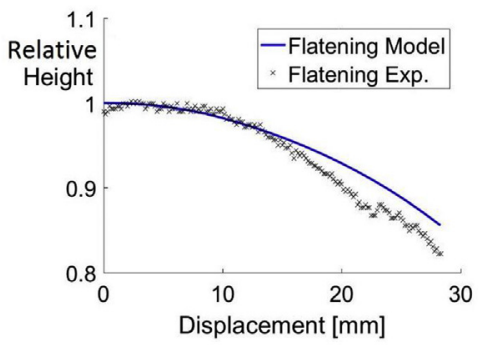

(a)

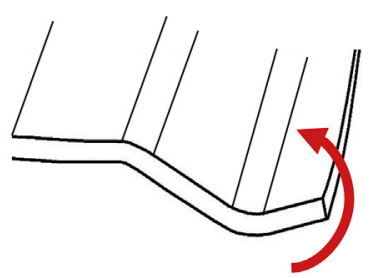

(b)
Fig. 11. (a) Experimental validation of the modelling results through height variation analysis for the pure SRPP panel and (b) the rotation of the crosssection under the loading nose. 
fractures occurred simultaneously. This effect tends to decrease the maximum flexural load compared to other layups, in which the layer fractures can occur independently. The height variation of the corrugated panel also contributes to the loss of load carrying capacity.

The tensile and compressive elastic moduli of SRPP are similar but the compressive strength is much lower than the tensile strength. In flexure, SRPP always fails under compression, which is evidenced by the flexural behaviour of the corrugated panels.

An FE model was developed to study the flexural properties of the panels. The experimental trends were confirmed by the model, although the model's predictions of the stiffness and failure force were systematically lower. Thickness variations in the corrugated panels and the increase in the volume fraction of the carbon fibre layers were possible causes for the difference.

When designing a hybrid corrugated panel made of SRPP and carbon fibres, a dispersed layup should be chosen over the stacking of multiple $\mathrm{CF}$ layers together, since the latter configuration reduces the maximum flexural load. For a composite panel with a threefold increase in stiffness against pure SRPP but still high toughness, one carbon fibre layer should be added in the middle. The developed FE model is also a useful tool to guide the design process, as it shows the trends in the stiffness and strength variation of the corrugated panels.

\section{Acknowledgments}

FM acknowledges the European Union for his Erasmus scholarship. YS acknowledges FWO Flanders for his postdoctoral fellowship. SVL acknowledges the Toray Chair.

\section{References}

[1] Schimanski T, Loos J, Peijs T, Alcock B, Lemstra PJ. On the overdrawing of meltspun isotactic poltpropylene tapes. J Appl Polym Sci 2007;103:2920-31. https:// doi.org/10.1002/app.25128.

[2] Ward IM, Hine PJ. The science and technology of hot compaction. Phys Lett B 2004:593:302-12. https://doi.org/10.1016/S0370-2693(04)00789-0.

[3] Alcock B, Peijs T. Technology and development of self-reinforced polymer composites. In: Abe A, Kausch H-H, Möller M, Pasch H, editors. Polym. Compos. - polyolefin fractionation - polym. Pept. - collagens Berlin, Heidelberg: Springer Berlin Heidelberg; 2013. p. 1-76. https://doi.org/10.1007/12_2011_159.

[4] Alcock B, Cabrera NO, Barkoula NM, Loos J, Peijs T. The mechanical properties of unidirectional all-polypropylene composites. Compos Part A Appl Sci Manuf 2006;37:716-26. https://doi.org/10.1016/j.compositesa.2005.07.002.

[5] Alcock B, Cabrera NO, Barkoula NM, Spoelstra AB, Loos J, Peijs T. The mechanical properties of woven tape all-polypropylene composites. Compos Part A Appl Sci Manuf 2007;38:147-61. https://doi.org/10.1016/j.compositesa.2006.01.003.

[6] Swolfs Y, Shi J, Meerten Y, Hine P, Ward I, Verpoest I, et al. The importance of bonding in intralayer carbon fibre/self-reinforced polypropylene hybrid composites. Compos Part A Appl Sci Manuf 2015;76:299-308. https://doi.org/10.1016/j. compositesa.2015.06.017.

[7] Jones RS, Riley DE. A new self-reinforced polypropylene composite. Proc 2nd annu conf soc plast eng automot compos div. 2002. p. 1-7. Michigan.

[8] Davies IJ, Hamada H. Flexural properties of a hybrid polymer matrix composite containing carbon and silicon carbide fibres. Adv Compos Mater 2001;10:77-96. https://doi.org/10.1163/15685510152546376.

[9] Giancaspro JW, Papakonstantinou CG, Balaguru PN. Flexural response of inorganic hybrid composites with E-glass and carbon fibers. J Eng Mater Technol 2010;132:021005. https://doi.org/10.1115/1.4000670.

[10] Dong C, Ranaweera-Jayawardena HA, Davies IJ. Flexural properties of hybrid composites reinforced by S-2 glass and T700S carbon fibres. Compos B Eng 2012;43:573-81. https://doi.org/10.1016/j.compositesb.2011.09.001.

[11] Zhang J, Chaisombat K, He S, Wang CH. Glass/carbon fibre hybrid composite laminates for structural applications. Sustain Automot Technol 2012:69-74. https:// doi.org/10.1007/978-3-642-24145-1_10.

[12] Yokozeki T, Takeda S ichi, Ogasawara T, Ishikawa T. Mechanical properties of corrugated composites for candidate materials of flexible wing structures. Compos Part A Appl Sci Manuf 2006;37:1578-86. https://doi.org/10.1016/j.compositesa. 2005.10.015.

[13] Samanta A, Mukhopadhyay M. Finite element static and dynamic analyses of folded plates. Eng Struct 1999;21:277-87. https://doi.org/10.1016/S0141-0296(97) 90172-3.

[14] Giroux C, Shao Y. Flexural and shear rigidity of composite sheet piles. J Compos Construct 2003;7:348-55. https://doi.org/10.1061/(ASCE)1090-0268(2003) $7: 4(348)$.

[15] Swolfs Y, De Cuyper P, Callens MG, Verpoest I, Gorbatikh L. Hybridisation of two ductile materials - steel fibre and self-reinforced polypropylene composites. Compos Part A Appl Sci Manuf 2017;100:48-54. https://doi.org/10.1016/j. compositesa.2017.05.001.

[16] Swolfs Y, Van Den Fonteyne W, Baets J, Verpoest I. Failure behaviour of self-reinforced polypropylene at and below room temperature. Compos Part A Appl Sci Manuf 2014:65:100-7. https://doi.org/10.1016/j.compositesa.2014.06.003.

[17] Swolfs Y, Zhang Q, Baets J, Verpoest I. The influence of process parameters on the properties of hot compacted self-reinforced polypropylene composites. Compos Part A Appl Sci Manuf 2014;65:38-46. https://doi.org/10.1016/j.compositesa.2014.05. 022.

[18] ASTM D 7264/D 7264M - 07. Standard test method for flexural properties of polymer matrix composite materials. 2007.

[19] ASTM D3410. Standard test method for compressive properties of polymer matrix composite materials with unsupported gage section by shear loading. 2003. [n.d].

[20] Tabatabaei SA, Swolfs Y, Wu H, Lomov SV. Full-field strain measurements and meso-FE modelling of hybrid carbon/self-reinforced polypropylene. Compos Struct 2015;132:864-73. https://doi.org/10.1016/j.compstruct.2015.07.018.

[21] Shindo A. Polyacrylonitrile (PAN)-based carbon fibers. Kelly A, Zweben C, Chou T, editors. Compr. Compos. Mater, vol. 1. Elsevier; 2000. p. 1-33.

[22] Searles K, Odegard G, Kumosa M. Micro- and mesomechanics of 8-harness satin woven fabric composites: I - evaluation of elastic behavior. Compos - Part A Appl Sci Manuf 2001;32:1627-55. https://doi.org/10.1016/S1359-835X(00)00181-0.

[23] T700S Data Sheet. Http://WwwToraycfaCom/Pdfs/T700SDataSheetPdf 2016:06-20. http://www.toraycfa.com/pdfs/T700SDataSheet.pdf (accessed June 20, 2016).

[24] Chamis CC. Simplified composite micromechanics equations for hygral, thermal and mechanical properties. NASA tech memo 8820. Houston: United States NASA; 1983.

[25] Pae KD, Chu H-C, Lee JK, Kim J-H. Healing of stress-whitening in polyethylene and polypropylene at or below room temperature. Polym Eng Sci 2000;40:1783-95. https://doi.org/10.1002/pen.11310.

[26] Swolfs Y, Crauwels L, Breda E Van, Gorbatikh L, Hine P, Ward I, et al. Tensile behaviour of intralayer hybrid composites of carbon fibre and self-reinforced polypropylene. Compos Part A Appl Sci Manuf 2014;59:78-84. https://doi.org/10. 1016/j.compositesa.2014.01.001. 